\title{
Combination therapy with S-1 and irinotecan (CPT-11) for advanced or recurrent gastric cancer
}

\author{
HiROYA TAKIUCHI \\ Cancer Chemotherapy Center, Osaka Medical College, 2-7 Daigaku-cho, Takatsuki, Osaka 569-8686, Japan
}

\begin{abstract}
S-1 and irinotecan (CPT-11) are active agents against gastric cancer. Some preclinical studies have demonstrated the theoretical background of combination therapy with S-1 and CPT11 for gastric cancer. Based on these findings, several phase I/II studies of this combination therapy, which has been proposed as a candidate of standard treatment for advanced or recurrent gastric cancer in Japan, have been conducted. Although there were slight differences in the administration schedules of the combination therapy with S-1 and CPT-11 in these phase II studies, the response rates were more than $50 \%$, and the median survival time (MST) exceeded 1 year. Also, good safety profiles were reported. These results warranted a further, phase III, study to define the efficacy of the combination in improving survival. In a phase III study (GC0301/TOP 002 trial), the response rate, the 1-year survival rate, and the MST in the arm with combination therapy of S-1 and CPT-11 were better than these parameters in the S-1 monotherapy arm. However, at 1.5-year follow-up, the overall survival (OS) in the combination therapy of S-1 and CPT-11 arm did not exceed that in the $S-1$ monotherapy $\operatorname{arm}[P=0.23$; hazard ratio (HR), 0.89]. As $22 \%$ of the patients were censored, further follow-up is needed to determine the OS with more precision. But, of note, in the SPIRITS trial, combination therapy with S-1 and cisplatin (CDDP) significantly prolonged OS compared to S-1 monotherapy $(P=0.037$; HR, 0.77), suggesting that CPT-11 may not be the best partner to use in combination with $\mathrm{S}-1$ at present.
\end{abstract}

Key words Gastric cancer · Irinotecan · S-1 · Combination chemotherapy

\section{Introduction}

S-1, an oral 5-fluorouracil (FU) derivative developed in Japan in 1999, consists of tegafur (FT) and two modula-

Offprint requests to: $\mathrm{H}$. Takiuchi

Received: February 6, 2008 / Accepted: April 13, 2008 tors, 5-chloro-2, 4-dihydroxypyridine [CDHP; a potent dihydropyrimidine dehydrogenase (DPD) inhibitor] and potassium oxonate (OXO; an orotate phosphoribosyl transferase inhibitor), at a molar ratio of 1:0.4:1, with the two modulators added in order to enhance antitumor effects via increasing the blood 5-FU concentration and reducing digestive toxicity $[1,2]$. Two late phase II studies demonstrated the efficacy of singleagent therapy with S-1 for gastric cancer; the response rates were $49 \%(25 / 51)$ and $44 \%$ (19/43), respectively; these rates being high for single-agent anticancer therapy $[3,4]$. In addition, S- 1 is an oral preparation, and this route may be advantageous for patients undergoing chemotherapy. Thus, in Japan, single-agent therapy with $\mathrm{S}-1$ has been emphasized as a standard treatment for advanced or recurrent gastric cancer. In 2007, the Japan Clinical Oncology Group (JCOG) presented the results of a randomized phase III study (JCOG 9912) including S-1 monotherapy at the annual meeting of the American Society of Clinical Oncology (ASCO); the efficacy of S-1 showed significant noninferiority to that of 5-FU alone. S-1 was recognized as a potent oral antitumor agent [5].

Irinotecan (CPT-11) is also an antitumor agent developed in Japan; its action mechanism involves the inhibition of topoisomerase I [6]. Concerning the efficacy of CPT-11 monotherapy for advanced gastric cancer, the response rates were $19.0 \%$ (4/21) and $18.4 \%$ (14/76), respectively, in early and late phase II studies in Japan $[7,8]$. This agent may be useful for treating advanced or recurrent gastric cancer. Various combination therapies incorporating CPT- 11 have been studied in clinical trials in Japan, especially combinations with cisplatin (CDDP) and S-1, and the potent antitumor effects of these combinations have been confirmed [9-13]. Based on these results, two randomized phase III studies were conducted in Japan. In this article, I focus on combination therapy with S-1 and CPT-11 for advanced or recurrent gastric cancer, and review the results of phase 
I/II clinical studies of combination therapy with S-1 and CPT-11 that were conducted in Japan [10-13]. In addition, I discuss the current and future perspectives of this combination therapy, based on the results of a randomized phase III study (S-1 vs S-1 + CPT-11; GC0301/TOP 002) reported at the Gastrointestinal Cancer Symposium in 2008 [14].

\section{Theoretical background of combination therapy with S-1 and CPT-11 in gastric cancer}

The action mechanism of fluoropyrimidines differs from that of CPT-11, and animal experimental studies have demonstrated the effects of fluoropyrimidine and CPT-11 combination therapy [15]. In colorectal cancer patients, combination therapy with CPT-11 and 5-FU (such as FOLFIRI regimen) was effective [16, 17]. In gastric cancer patients, the combination of fluoropyrimidines and CPT-11 may also be useful. Takiuchi et al. [18] reported that S-1 plus CPT-11 showed an augmented antitumor effect against 5-FU-resistant tumors with high thymidylate synthase (TS) activity, compared to CPT-11 alone and S-1 alone in an experimental study. A potential mechanism of this effect was suggested by the significant reduction in TS activity observed in tumors with high TS activity following CPT-11 administration (Fig. 1) [18]. Ichikawa et al. [19] investigated the mRNA expression of TS and a 5-FUmetabolizing enzyme, DPD, in their series, and indicated that many patients with low-level TS expression responded to S-1 alone, whereas a high proportion of patients with S-1-resistant tumors (some of whom had high-level TS-expression) responded to the combination of S-1 and CPT-11. On the other hand, both
Takiuchi et al. [18] and Ichikawa et al. [19] reported that there was no relationship between high or low DPD mRNA expression and tumor response to either S-1 monotherapy or to combination therapy with S-1 and CPT-11. Fluoropyrimidines are a mainstay of palliative treatment for advanced or recurrent gastric cancer. S-1 has a theoretical advantage over 5-FU in terms of having antitumor activity that appears to be independent of the level of DPD expression, suggesting that $\mathrm{S}-1$ should be active in tumors expected to be resistant to 5-FU on the basis of high DPD expression. Moreover, the combination of S-1 and CPT- 11 should also be active in tumors expected to be resistant to S-1 monotherapy on the basis of high TS expression. Based on this theoretical background, several phase I/II clinical studies of combination therapy with S-1 and CPT-11 were conducted.

\section{Phase I/II clinical studies of combination therapy with S-1 and CPT-11}

The results of the main phase I/II studies of combination therapy with S-1 and CPT-11 in patients with advanced or recurrent gastric cancer in Japan are summarized in Table 1. Komatsu et al. [10] conducted a phase I study to estimate the maximum tolerated dose (MTD) of CPT-11 on days 1 and 15, given in combination with S-1, administered at a fixed dose of $40 \mathrm{mg} / \mathrm{m}^{2}$ twice daily on days 1-14 of each 4-week cycle. Fifteen patients were treated with increasing dose levels of CPT-11, as follows: CPT-11, $100 \mathrm{mg} / \mathrm{m}^{2}$ (level 1); $125 \mathrm{mg} / \mathrm{m}^{2}$ (level 2); and $150 \mathrm{mg} / \mathrm{m}^{2}$ (level 3). All the patients were found to be assessable for drug safety. If level 3 was tolerated, this dose became the

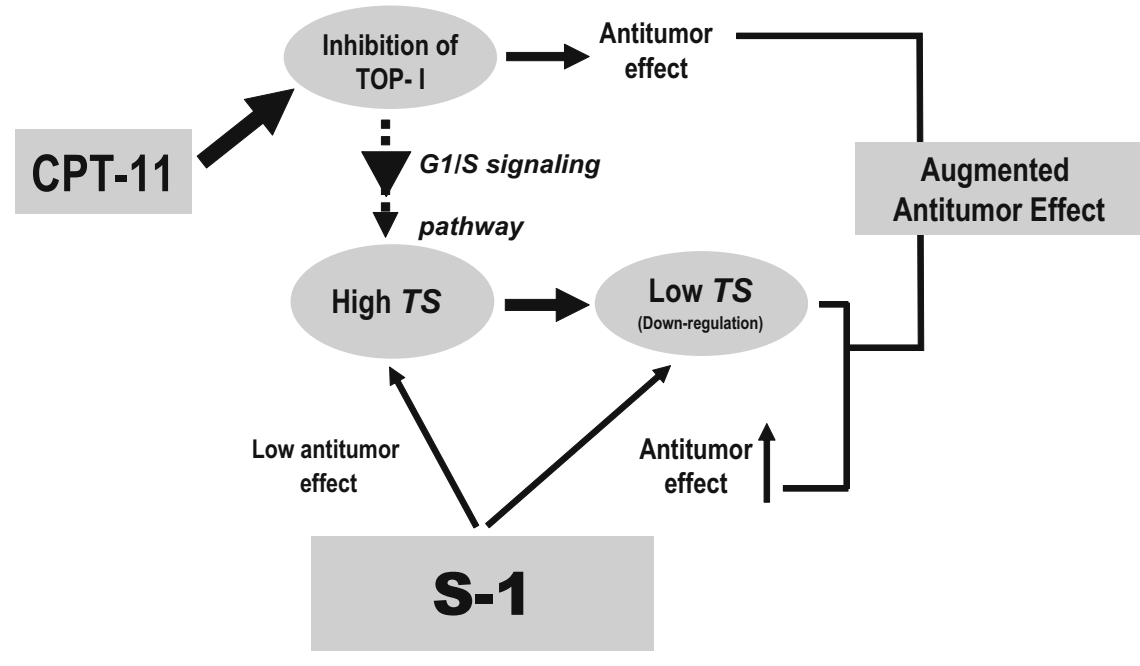

Fig. 1. Possible interaction between irinotecan $(C P T-11)$ and $\mathrm{S}-1$, which consists of tegafur and two modulators, 5-chloro2, 4-dihydroxypyridine (CDHP; a potent dihydropyrimidine dehydrogenase inhibitor), and potassium oxonate (OXO; an orotate phosphoribosyl transferase inhibitor), at a molar ratio of 1: 0.4: 1 . TOP-I, Topoisomerase I; TS, thymidylate synthase 


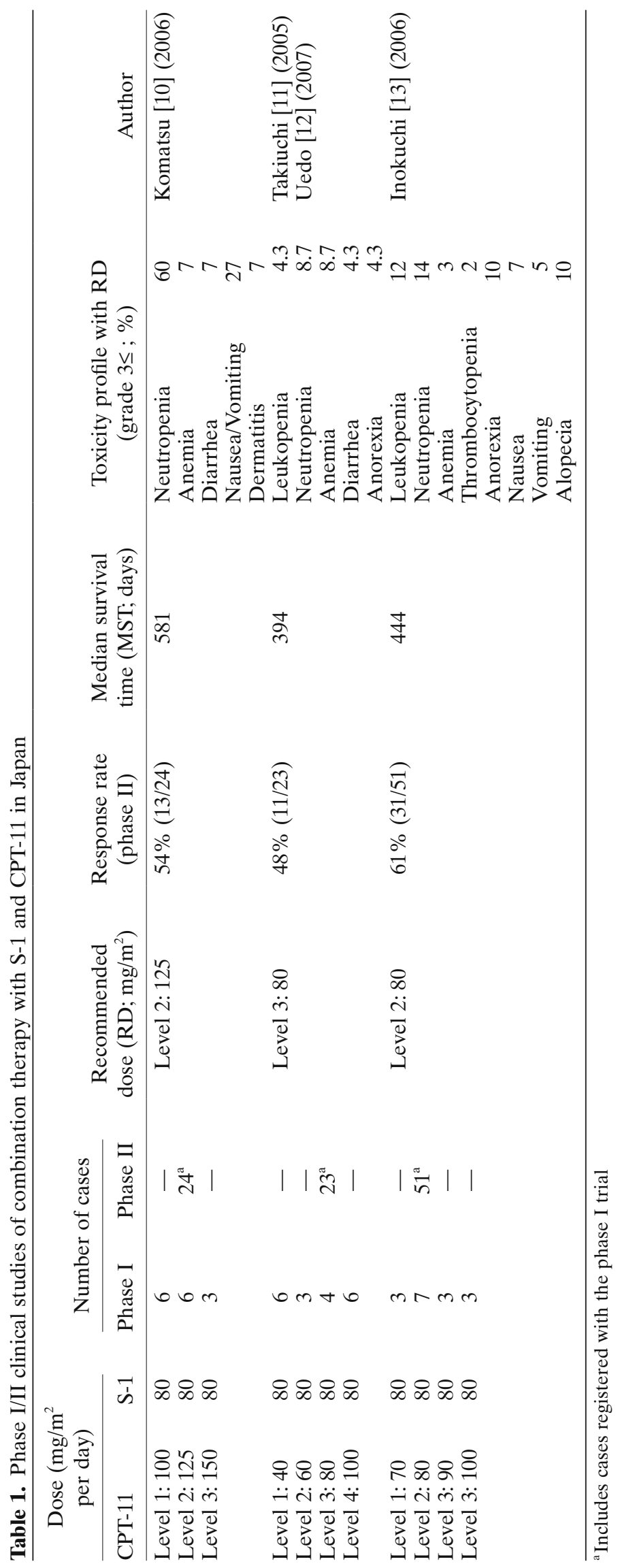


recommended dose in combination with $\mathrm{S}-1$, because the maximum approved dose of CPT-11 alone in Japan is $150 \mathrm{mg} / \mathrm{m}^{2}$. S-1, with dosage determined on the basis of the body surface area (BSA), was given orally for 2 weeks at the following doses: $40 \mathrm{mg}\left(\mathrm{BSA}<1.25 \mathrm{~m}^{2}\right)$, $50 \mathrm{mg}\left(\mathrm{BSA} \geq 1.25\right.$ to $\left.<1.5 \mathrm{~m}^{2}\right)$, or $60 \mathrm{mg}\left(\mathrm{BSA} \geq 1.5 \mathrm{~m}^{2}\right)$ twice daily (standard dose in Japan), followed by 2 weeks without treatment. Even at level 3, the MTD was not reached. However, at level 1, marked bone marrow suppression was noted in 1 patient. Therefore, the Efficacy and Safety Committee recommended that the recommended dose (RD) of CPT-11 should be established as $125 \mathrm{mg} / \mathrm{m}^{2}$. In a phase II study, 24 patients with measurable lesions were enrolled. A total of 91 cycles were administered (median, 5.9 cycles). The response rate was $54 \%$ (13/24), and the median survival time (MST) was 581 days. The main grade 3 or higher side effects included neutropenia $(60 \%)$ and nausea/vomiting $(27 \%)$.

Takiuchi et al. [11] conducted a phase I study to determine the MTD and RD of CPT-11, given on days 1 and 15 in combination with S-1 administered at a fixed dose of $40 \mathrm{mg} / \mathrm{m}^{2}$ twice daily on days $1-21$ of each 5week cycle. Nineteen patients were treated with increasing dose levels of CPT-11, as follows: CPT- $11,40 \mathrm{mg} / \mathrm{m}^{2}$ (level 1); $60 \mathrm{mg} / \mathrm{m}^{2}$ (level 2); $80 \mathrm{mg} / \mathrm{m}^{2}$ (level 3); and $100 \mathrm{mg} / \mathrm{m}^{2}$ (level 4). No dose-limiting toxicity (DLT) was observed at either level 1,2, or 3. At dose level 4, three of six patients exhibited DLTs in the first course; one of the three patients had grade 3 diarrhea, and the other two patients had grade 3 rash; all three skipped the second administration of CPT- 11 on day 15 because of delayed resolution of grade 2 leukopenia. The RD of CPT-11 in combination with S-1 was the dose of $80 \mathrm{mg} /$ $\mathrm{m}^{2}$ at level 3 according to the protocol definitions [11]. In a phase II study, 23 patients were enrolled to investigate the efficacy and safety of the combination. The response rate, median time to progression (TTP), and MST were 47.8\% (11/23), 210 days, and 394 days, respectively. The incidence of grade 3 or 4 hematological and nonhematological toxicities was $17.4 \%$ and $8.4 \%$; the toxicities included neutropenia (8.7\%), anemia $(8.7 \%)$, diarrhea $(4.3 \%)$, anorexia $(4.3 \%)$, and nausea/vomiting ( $4.3 \%)$; the incidences of severe toxicities were generally low [12].

Inokuchi et al. [13] conducted a phase I study to estimate the MTD of CPT-11, given on days 1 and 8 in combination with $\mathrm{S}-1$ administered at a fixed dose of $40 \mathrm{mg} / \mathrm{m}^{2}$ twice daily on days $1-14$, followed by 2 weeks' rest. The cycle was repeated every 4 weeks. The doses of CPT-11 investigated were $70,80,90$, and $100 \mathrm{mg} / \mathrm{m}^{2}$. At $100 \mathrm{mg} / \mathrm{m}^{2}$, DLT was noted in two of three patients. Initially, the RD was established as $90 \mathrm{mg} / \mathrm{m}^{2}$. According to subsequent follow-up data, grade 4 bone marrow suppression was observed at $90 \mathrm{mg} / \mathrm{m}^{2}$ in all three patients in the second course. Therefore, the RD was finally established as $80 \mathrm{mg} / \mathrm{m}^{2}$. In a phase II study, 51 patients were registered to estimate the efficacy and safety of the CPT-11 and S-1 combination. The response rate and MST were $61 \%(31 / 51)$ and 444 days, respectively. Grade 3 or higher side effects were neutropenia $(14 \%)$, anorexia $(10 \%)$, nausea $(7 \%)$, and vomiting (5\%) [13].

Although there were slight differences in the administration schedules, these phase II studies showed response rates of more than $50 \%$ and MSTs exceeding 1 year when a CPT- 11 and S- 1 combination was given as first-line treatment in patients with advanced or recurrent gastric cancer. In terms of toxicity profiles, the administration schedule employed by Takiuchi et al. [11] was considered to be more acceptable than the schedules reported by Komatsu et al. [10] and Inokuchi et al. [13]. Based on these results, a randomized phase III trial has been conducted to evaluate the efficacy of combination therapy with S-1 and CPT- 11 in improving survival, compared with that of S-1 monotherapy, for advanced or recurrent gastric cancer (GC0301/TOP 002).

\section{Current status and future perspectives of combination therapy with S-1 and CPT-11}

The results of the GC0301/TOP 002 (S-1 vs S-1 + CPT11) trial were announced by Imamura et al. [14] at the Gastrointestinal Cancer Symposium in 2008. The primary endpoint was overall survival (OS), and the secondary endpoints were time to treatment failure, 1year survival rate, response rate, and safety. The followup period was 1.5 years. In patients evaluable according to the response evaluation criteria in solid tumors (RECIST), the response rates were $26.9 \%$ in the S-1 monotherapy arm and $41.5 \%$ in the combination therapy of S-1 and CPT-11 arm, with the difference being statistically significant $(P=0.035)$. The 1 -year survival rate was $44.9 \%$ in the S-1 monotherapy arm and $52.0 \%$ in the combination therapy of S-1 and CPT- 11 arm. In the two arms, the main grade 3 or higher side effects consisted of neutropenia (S-1 vs S-1 + CPT-11; $10.6 \%$ vs $26.6 \%$ ), diarrhea (S-1 vs S- $1+\mathrm{CPT}-11 ; 5.6 \%$ vs $15.8 \%$ ), anorexia (S-1 vs S-1 + CPT-11; 9.9\% vs $15.8 \%$ ), nausea (S-1 vs S-1 + CPT-11; $3.7 \%$ vs $7.0 \%$ ), and vomiting (S-1 vs $\mathrm{S}-1+\mathrm{CPT}-11 ; 0.6 \%$ vs $2.5 \%$ ). The incidences of severe toxicity were slightly higher in the combination of S-1 and CPT-11 arm, but the toxicity was tolerable in both arms. The MST in the S-1 monotherapy arm was 318 days and that in the combination of S- 1 and CPT-11 arm was 389 days; however, at 1.5-year follow up, the OS in the combination of S-1 and CPT-11 arm did not significantly exceed that in the S-1 monotherapy arm $[P$ 
$=0.23$; hazard ratio $(\mathrm{HR})=0.89]$. It was concluded that, considering that 68 patients $(22 \%)$ were censored (mainly due to unexpectedly long survival times in both arms), further follow-up would be needed to confirm the OS with more precision.

On the other hand, as it was announced - at the annual meeting of ASCO in 2007 (SPIRITS trial) that combination therapy with S-1 and CDDP significantly prolonged OS compared to S-1 monotherapy, it seems that CPT-11 may not be the best partner in combination with S-1 at present [20]. In a previous study comparing CPT-11 plus 5-FU/folinic acid (IF) with 5FU plus CDDP (FP), OS in the IF arm did not exceed that in the FP arm, leading to the conclusion that IF would be the treatment choice for patients in whom CDDP administration is not possible for some reason [21]. In addition, in the JCOG 9912 trial reported by Boku et al. [5] in 2007, at the annual ASCO meeting, combination therapy with CPT-11 and CDDP did not show statistically significant superiority to 5-FU monotherapy, in terms of OS. Therefore, at present, no CPT11-based regimens can be recommended as first-line treatment for advanced or recurrent gastric cancer. However, the report of the GC0301/TOP002 trial is not a final result, and further follow-up of the censored cases is still underway. In the near future, the final report of this trial should help us to resolve the question of the use of CPT-11 for treating gastric cancer.

\section{References}

1. Fukushima M, Satake H, Uchida J, Shimamoto Y, Kato T, Takechi T, et al. Preclinical antitumor efficacy of S-1: a new oral formulation of 5-fluorouracil on human tumor xenografts. Int J Oncol 1998;13:693-8.

2. Shirasaka T, Nakano K, Takechi T, Satake H, Uchida J, Fujioka A, et al. Antitumor activity of $1 \mathrm{M}$ tegafur-0.4M 5-chloro-2,4dihydroxypyridine-1M potassium oxonate (S-1) against human colon carcinoma orthotopically implanted into nude rats. Cancer Res 1996;56:2602-6.

3. Koizumi W, Kurihara M, Nakano S, Hasegawa K. Phase II study of S-1, a novel oral derivative of 5-fluorouracil, in advanced gastric cancer. For the S-1 Cooperative Gastric Cancer Study Group. Oncology 2000;58:191-7.

4. Sakata Y, Ohtsu A, Horikoshi N, Sugimachi K, Mitachi Y, Taguchi T. Late phase II study of novel oral fluoropyrimidine anticancer drug S-1 (1M tegafur- $0.4 \mathrm{M}$ gimestat- $1 \mathrm{M}$ otastat potassium) in advanced gastric cancer patients. Eur J Cancer 1998;34: 1715-20.

5. Boku N, Yamamoto S, Shirao K, Doi T, Sawaki A, Koizumi W, et al. Randomized phase III study of 5-fluorouracil (5-FU) alone versus combination of irinotecan and cisplatin (CP) versus S-1 alone in advanced gastric cancer (JCOG 9912). Proc Am Soc Clin Oncol 2007;25:4513.

6. Kawato Y, Aonuma M, Hirota Y, Kuga H, Sato K. Intracellular roles of SN-38, a metabolite of the camptothecin derivative CPT-
11, in the antitumor effect of CPT-11. Cancer Res 1991;51: 4187-91.

7. Sakata Y, Nakao I, Futatsuki K, Kambe M, Wakui A, Taguchi T. An early phase II trial of CPT-11 in patients with advanced gastrointestinal cancer (in Japanese with English abstract). J Jpn Soc Cancer Ther 1992;27:2028-35.

8. Futatsuki K, Wakui A, Nakao I, Sakata Y, Kambe M, Shimada Y, et al. Late phase II study of irinotecan hydrochloride (CPT-11) in advanced gastric cancer (in Japanese with English abstract). Jpn J Cancer Chemother 1994;21:1033-8.

9. Boku N, Ohtsu A, Shimada Y, Shirao K, Seki S, Saito H, et al. Phase II study of a combination of irinotecan and cisplatin against metastatic gastric cancer. J Clin Oncol 1999;17:319-23.

10. Komatsu Y, Yuki S, Miyagishima T, Asaka M. Irinotecan plus oral S-1 in patients with advanced gastric cancer-biweekly IRIS regimen (in Japanese with English abstract). Gan To Kagaku Ryoho (Jpn J Cancer Chemother) 2006;33(Suppl 1):75-8.

11. Takiuchi H, Narahara H, Tsujinaka T, Gotoh M, Kawabe S, Katsu K, et al. Osaka Gastrointestinal Cancer Chemotherapy Study Group (OGSG). Phase I study of S-1 combined with irinotecan (CPT-11) in patients with advanced gastric cancer (OGSG 0002). Jpn J Clin Oncol 2005;35:520-5.

12. Uedo N, Narahara H, Ishihara R, Takiuchi H, Goto M, Fujitani $\mathrm{K}$, et al. Phase II study of a combination of irinotecan and S-1 in patients with advanced gastric cancer (OGSG0002). Oncology 2007;73:65-71.

13. Inokuchi M, Yamashita T, Yamada H, Kojima K, Ichikawa W, Nihei Z, et al. Phase I/II study of S-1 combined with irinotecan for metastatic advanced gastric cancer. $\mathrm{Br} \mathrm{J}$ Cancer 2006;94: $1130-5$.

14. Imamura $H$, Iishi $H$, Tsuburaya $A$, Hatake $\mathrm{K}$, Imamoto $H$, Esaki $\mathrm{M}$, et al. Randomized phase III study of irinotecan plus S-1 (IRIS) versus S-1 alone as first-line treatment for advanced gastric cancer (GC0301/TOP-002). 2008 Gastrointestinal Cancer Symposium Abstract \#5, January 2008, Orland, USA.

15. Cao S, Rustum YM. Synergistic antitumor activity of irinotecan in combination with 5-fluorouracil in rats bearing advanced colorectal cancer: role of drug sequence and dose. Cancer Res 2000;60:3717-21.

16. Douillard JY, Cunningham D, Roth AD, Navarro M, James RD, Karasek P, et al. Irinotecan combined with fluorouracil compared with fluorouracil alone as first-line treatment for metastatic colorectal cancer: a multicentre randomised trial. Lancet 2000; 355:1041-7.

17. Saltz LB, Cox JV, Blanke C, Rosen LS, Fehrenbacher L, Moore $\mathrm{MJ}$, et al. Irinotecan plus fluorouracil and leucovorin for metastatic colorectal cancer. Irinotecan Study Group. N Engl J Med 2000;343:905-14.

18. Takiuchi H, Kawabe S, Gotoh M, Katsu K. Thymidylate synthase gene expression in primary tumors as predictive parameters for the efficacy of S-1-based chemotherapy for advanced gastric cancer. Gastrointestinal Cancer Res 2007;1:172-7.

19. Ichikawa W, Takahashi T, Suto K, Shirota Y, Nihei Z, Shimizu $\mathrm{M}$, et al. Thymidilate synthase predictive power is overcome by irinotecan combination therapy with S-1 for gastric cancer. Br J Cancer 2004;91:1245-50.

20. Narahara H, Koizumi W, Hara T, Takagane A, Akiya T, Takagi M, et al. Randomized phase III study of S-1 alone versus $\mathrm{S}-1+$ cisplatin in the treatment for advanced gastric cancer (the SPIRITS trial). Proc Am Soc Clin Oncol 2007;25:4514.

21. Dank M, Zaluski J, Barone C, Valvere V, Peschel C, Wenczl M, et al. Randomized phase 3 trial of irinotecan (CPT-11) + 5-FU/ folinic acid (FA) vs CDDP + 5-FU in first-line advanced gastric cancer patients. Proc Am Soc Clin Oncol 2005;23:4003. 